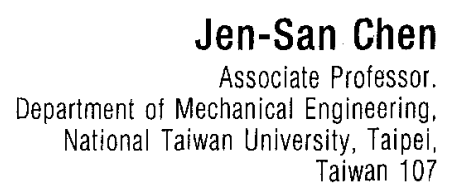

Taiwan 107

\title{
Natural Frequencies and Stability of an Axially-Traveling String in Contact With a Stationary Load System
}

The natural frequencies and stability of a string traveling between two fixed supports and in contact with a stationary load system, which contains such parameters as dry friction, inertia, damping, and stiffness, are investigated both numerically and analytically. After establishing the orthogonality properties between the eigenfunctions of a freely traveling string, the eigenvalues of the coupled system are calculated by a numerical procedure based on eigenfunction expansion method. It is found that the stiffness in the load system tends to increase the natural frequencies of the traveling string, while the inertia tends to decrease the natural frequencies. When the load system contains both inertia and stiffness elements with natural frequency $\omega_{z}$, inertia effect is dominant for the modes with natural frequencies higher than $\omega_{z}$, and stiffness effect is dominant for the modes with natural frequencies lower than $\omega_{z}$. Large dry friction causes flutter instability in the high speed range, which cannot be suppressed by the damping element in the load system. The expressions for the derivatives of eigenvalues with respect to various load parameters are derived to verify the numerical results.

\section{Introduction}

The dynamics of axially-traveling strings has been a research topic of interest because of its important applications in such fields as magnetic and paper tapes, textile fibers, aerial cable tramways, power transmission chains, and band saws. Excessive vibration of axially traveling structure is generally undesirable. In magnetic tape drives, vibration leads to signal modulation and accelerated wear of the tape. In band saws, the transverse vibration of the blade results in poor cutting quality. Following Skutch's (1897) work, extensive literature has been devoted to the study of linear, planar vibration and stability of a string moving between two fixed supports. Comprehensive survey and fairly complete references on this subject can be found in the review paper by Wickert and Mote (1988).

Early investigations focus on aspects of free vibration including the nature of wave propagation in the moving string (Sack, 1954; Mahalingam, 1957; Miranker, 1960; Swope and Ames, 1963 ) and the relations between the system natural frequencies and the traveling speed of the string (Archibald and Emslie, 1958 ). The natural frequencies of the translating string decrease monotonically as the traveling speed increases. The corresponding mode shapes are transverse waves traveling upstream within a stationary envelope (Rhodes, 1970). At certain traveling speed, called critical speed in the literature, all the natural frequencies reduce to zero and the associated stiffness operator in the equation of motion becomes singular. Hwang and Perkins (1992a, b) studied the vibration and stability of an axially moving beam in the supercritical speed range with nonlinear formulation. They observed bifurcation phenomena and multiple equilibria in the supercritical speed range with the traveling speed as a control parameter, which are similar to the buckling instability of a column with axial compressive loading as a control parameter.

Contributed by the Technical Committee on Vibration and Sound for publication in the Journal of Vibration AND ACOUSTICS. Manuscript received June 1994. Associate Technical Editor: K. W. Wang.
In some applications the traveling string may interact with a stationary load system. This stationary load system may represent the read/write head in magnetic tape drives, or the guide pad in band saws. Schajer (1984) studied the dynamic response of a rotating circular string subject to a fixed elastic constraint, with an aim toward demonstrating the phenomenon of eigenvalue "veering" often encountered in complex rotating systems. Cheng and Perkins (1991) studied the stability of a traveling string subject to large dry friction and reported that dry friction induces flutter instability in the high speed range. Often the inertia of the stationary load system is small and may be neglected, as have been done in the above two papers. However, there are some problems of physical importance in which load inertia is not negligible and may alter the dynamic behavior of the system significantly. Besides, the role of the damping element in the load system in stabilizing the traveling string is not clear. A thorough investigation dealing with the interaction between the axially-traveling string and various dynamic elements in the stationary load system is still not available in the literature.

In the present paper we consider a string traveling between two fixed supports and in contact with a stationary load system, which contains such parameters as dry friction, inertia, damping, and stiffness. The dimensionless equation of motion is first cast in first-order form and the orthogonality properties are established. The effects of various load parameters on the natural frequencies and stability of the system are then investigated through a numerical procedure based on eigenfunction expansion method. The derivatives of eigenvalues with respect to various load parameters are also derived to verify the numerical results.

\section{Equation of Motion}

Consider a uniform, flexible string of linear mass density $\rho$ that travels with speed $V$ between two fixed frictionless eyelets which are separated by distance $L$. The string slides through an elastically supported guide at axial position $X=X_{0}$, where $X W$ coordinate system is fixed in space, as shown in Fig. 1 . The 


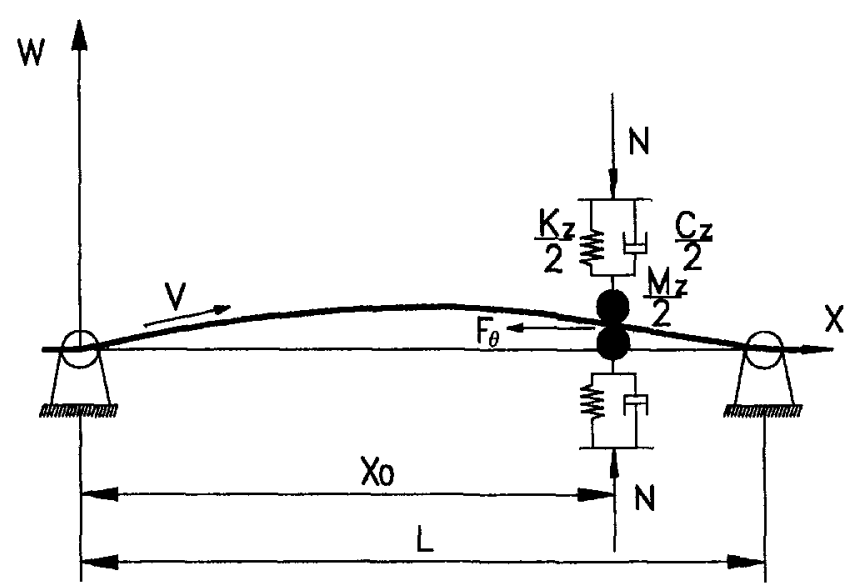

Fig. 1 A traveling string in contact with a stationary load system

top and bottom parts of the guide are assumed to be identical and can be modeled as spring-mass-damper systems with parameters $M_{z} / 2, K_{z} / 2$, and $C_{z} / 2$, respectively. The top and bottom surfaces of the guide are assumed to remain in contact with the string due to the relatively large normal force $N$ applied externally, and the resultant dry friction $F_{\theta}$ developed on the surfaces of the guide is equal to $2 \mu N$, where $\mu$ is the coefficient of friction. The string is subject to a constant tension $P$ to the right of $X$ $=X_{0}$, and is subject to $P-F_{\theta}$ to the left. The linearized equation of motion of this coupled system, in terms of transverse displacement $W$ and with respect to the stationary coordinate system, can be written as

$$
\begin{aligned}
& \rho\left(W_{, T T}+2 V W_{, X T}+V^{2} W_{, X X}\right)-F W_{, X X} \\
& \quad=-\left(K_{z} W+C_{z} W_{, T}+M_{z} W_{, T T}-F_{\theta} W_{, X}\right) \delta^{*}\left(X-X_{0}\right)
\end{aligned}
$$

where

$$
F= \begin{cases}P & \text { when } \quad X_{0}<X<L \\ P-F_{\theta} & \text { when } \quad 0<X<X_{0}\end{cases}
$$

The standard subscript notation for partial derivative is used here. $\delta^{*}(:)$ is the Dirac delta function.

After introducing the following definitions

$$
\begin{gathered}
x=\frac{X}{L}, \quad w=\frac{W}{L}, \quad t=T \sqrt{\frac{P}{\rho L^{2}}}, \nu=V \sqrt{\frac{\rho}{P}}, \\
\delta(x-\xi)=L \delta^{*}\left(X-X_{0}\right) \\
k_{z}=\frac{K_{z} L}{P}, \quad c_{z}=\frac{C_{z}}{\sqrt{\rho P}}, \quad m_{z}=\frac{M_{z}}{\rho L}, \quad f_{\theta}=\frac{F_{\theta}}{P},
\end{gathered}
$$

Equation (1) can be nondimensionalized and rewritten in the operator form

$$
(M+\hat{M}) w_{t, t}+(G+\hat{G}) w_{, t}+(K+\hat{K}) w=0
$$

where

$$
\begin{gathered}
M=1, \quad G=2 \nu \frac{\partial}{\partial x}, \quad K=\left(\nu^{2}-1\right) \frac{\partial^{2}}{\partial x^{2}} \\
\hat{M}=m_{z} \delta(x-\xi), \quad \hat{G}=c_{z} \delta(x-\xi) \\
\hat{K}=\delta(x-\xi)\left(k_{z}-f_{\theta} \frac{\partial}{\partial x}\right)+f_{\theta}[H(x)-H(x-\xi)] \frac{\partial^{2}}{\partial x^{2}}
\end{gathered}
$$

$H(:)$ is the Heaviside function. It is noted that friction force $f_{\theta}$ affects the natural frequencies and stability of the system through two separate terms in the operator $\hat{K}$. One is the term with Dirac delta function, which is associated with the slope change of the deformed string at the coupling point. The other is the term with Heaviside function, which is associated with the tension variation along the length of the string.

Equation (2) can also be cast in first-order operator form

$$
(\mathbf{A}+\hat{\mathbf{A}}) x_{,}-(\mathbf{B}+\hat{\mathbf{B}}) x=0
$$

by defining the state vector

$$
\mathbf{x}=\left\{\begin{array}{c}
w_{, t} \\
w
\end{array}\right\}
$$

and the matrix differential operators

$$
\begin{array}{cc}
\mathbf{A}=\left[\begin{array}{cc}
M & 0 \\
0 & K
\end{array}\right], \quad \hat{\mathbf{A}}=\left[\begin{array}{cc}
\hat{M} & 0 \\
0 & \hat{K}
\end{array}\right], \\
\mathbf{B}=\left[\begin{array}{cc}
-G & -K \\
K & 0
\end{array}\right], \quad \hat{\mathbf{B}}=\left[\begin{array}{cc}
-\hat{G} & -\hat{K} \\
\hat{K} & 0
\end{array}\right] .
\end{array}
$$

For a freely traveling string (i.e., in the absence of the load system), Eq. (2) can be reduced to

$$
M w_{, t t}+G w_{, t}+K w=0
$$

Since $M$ and $K$ are symmetric and $G$ is skew-symmetric, Eq. (4) is a standard gyroscopic equation. The eigenvalues of the $e^{\lambda t}$ time-reduced form of Eq. (4), together with the homogeneous boundary conditions, are purely imaginary and occur in complex conjugate pairs,

$$
\lambda_{n}^{0}=i \omega_{n}=\operatorname{in} \pi\left(1-\nu^{2}\right), \quad n=\ldots,-2,-1,1,2, \ldots
$$

where $i=\sqrt{-1} \cdot \omega_{n}$ is a real number and represents the natural frequency of the traveling string. The eigenfunction corresponding to $\lambda_{n}^{0}$ is in general complex and assumes the form

$$
w_{n}^{0}(x)=\sin (n \pi x) e^{i n \pi v x}
$$

The eigenfunction corresponding to $\bar{\lambda}_{n}^{0}$ is $\bar{w}_{n}^{0}$, where overbar represents complex conjugate. In the sub-critical speed range, i.e., $\nu<1$, the corresponding mode shape in Eq. (6) is a transverse wave traveling upstream within a stationary envelope. This property was also reported by Rhodes in 1970 .

Sometimes it is desirable to consider the mode shape in the real domain instead of the complex domain as implied in Eq. (6). In this way of thinking, the complete modal response for the $n$th natural frequency should be a combination of $w_{n}^{0}$ and $\bar{w}_{n}^{0}$, each of which contains both real and imaginary parts. The exact contributions from these two eigenfunctions depend on the initial modal displacement and velocity fields of the string. With this arrangement, the imaginary part will disappear naturally and only the real part (which is physically meaningful) of the modal response exists. For a traveling string with an initial displacement in its $n$th mode, the combination of these two eigenfunctions will naturally arrive to a traveling wave form.

In the case when the string travels in the supercritical speed range, the corresponding mode shape appears to be a wave traveling downstream. It is noted that the contribution of geometric non-linearity increases with translating speed, and the linear solution in the supercritical speed range is interpreted as a first approximation only (Hwang and Perkins, 1992a, b).

\section{Orthogonality Relations and Eigenfunction Expansion Method}

The orthogonality relations among the eigenfunctions of a gyroscopic system, when the stiffness operator $K$ in Eq. (4) is positive definite, have been established by Meirovitch (1974), D'Eleuterio and Hughes (1984), and Wickert and Mote (1990) 


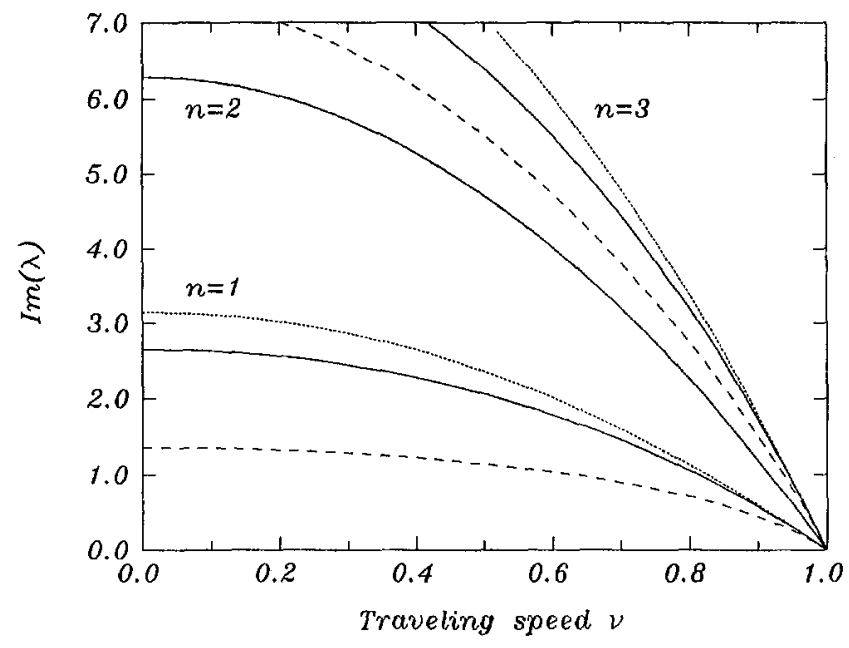

Fig. 2 Effects of inertia $m_{z}=0.2$ (solid lines) and 2 (dashed lines) at $\xi$ $=0.5$ on the natural frequencies of the traveling string, compared to the results for a freely traveling string (dotted lines). All other parameters are zero.

in various forms. Chen and Bogy (1992) extended the validity of these theorems to the case when $K$ is no longer positive definite. They showed that as long as all the eigenvalues of the gyroscopic system are purely imaginary, the following orthogonality relations exist:

$$
\left\langle\mathbf{x}_{m}^{0}, \mathbf{A} \mathbf{x}_{n}^{0}\right\rangle=0, \quad\left\langle\mathbf{x}_{m}^{0}, \mathbf{B} \mathbf{x}_{n}^{0}\right\rangle=0 \quad \text { if } \quad \lambda_{m}^{0} \neq \lambda_{n}^{0}
$$

where the inner product between two vectors $\mathbf{x}_{m}$ and $\mathbf{x}_{n}$ is defined as

$$
\left\langle\mathbf{x}_{m}, \mathbf{x}_{n}\right\rangle=\int_{0}^{1} \mathbf{x}_{m}^{T} \mathbf{x}_{n} d x
$$

$\mathbf{x}_{m}^{T}$ is the transpose of the state vector $\mathbf{x}_{m}$. In addition,

$$
\lambda_{n}^{0}=\frac{\left\langle\mathbf{x}_{n}^{0}, \mathbf{B} \mathbf{x}_{n}^{0}\right\rangle}{\left\langle\mathbf{x}_{n}^{0}, \mathbf{A} \mathbf{x}_{n}^{0}\right\rangle}
$$

To obtain the eigenvalues of Eq. (2) or (3) for the stringload system, we represent its eigenfunction solution as an expansion in terms of $2 \mathrm{~N}$ eigenfunctions of the freely traveling string,

$$
\mathbf{x}=\sum_{n=-N}^{N} h_{n} \mathbf{x}_{n}^{0}
$$

where, $h_{n}$ are complex numbers. It is noted that modes $\mathbf{x}_{n}^{0}$ and $\mathbf{x}_{-n}^{0}$ are complex conjugate pairs, and are orthogonal in the complex inner product space with inner product definition (8). Substituting Eq. (10) into (3) and taking the inner product between $\mathbf{x}_{m}^{0}$ and both sides of Eq. (3), with use of the orthogonality properties (7) and Eq. (9), we get

$$
\lambda \sum_{n=-N}^{N}\left(\hat{A}_{n}^{m}+A_{n}^{m}\right) h_{n}-\sum_{n=-N}^{N}\left(\hat{B}_{n}^{m}+\lambda_{n}^{0} A_{n}^{m}\right) h_{n}=0
$$

where

$$
\begin{aligned}
& A_{n}^{m}=0 \text { when } m \neq n \text { and } A_{m}^{m}=4 m^{2} \pi^{2}\left(1-\nu^{2}\right) \\
& \hat{A}_{n}^{m}=\left(\omega_{m} \omega_{n} m_{z}+k_{z}\right) \bar{w}_{m}^{0}(\xi) w_{n}^{0}(\xi) \\
& +f_{\theta}\left\{\int_{0}^{\xi} \bar{w}_{m}^{0} \frac{\partial^{2} w_{n}^{0}}{\partial x^{2}} d x-\left.\bar{w}_{m}^{0}(\xi) \frac{\partial w_{n}^{0}}{\partial x}\right|_{x=\xi}\right\}
\end{aligned}
$$

$$
\begin{aligned}
\hat{B}_{n}^{m}=[ & \left.-\omega_{m} \omega_{n} c_{z}+i k_{z}\left(\omega_{m}+\omega_{n}\right)\right] \bar{w}_{m}^{0}(\xi) w_{n}^{0}(\xi) \\
& +i f_{\theta}\left(\omega_{m}+\omega_{n}\right)\left\{\int_{0}^{\epsilon} \bar{w}_{m}^{0} \frac{\partial^{2} w_{n}^{0}}{\partial x^{2}} d x-\left.\bar{w}_{m}^{0}(\xi) \frac{\partial w_{n}^{0}}{\partial x}\right|_{x=\xi}\right\}
\end{aligned}
$$

Equation (11) represents a generalized eigenvalue problem with both matrices being complex. The eigenvalues can be readily extracted by any available eigenvalue solver.

\section{Numerical Results}

Figure 2 shows the relation between the natural frequencies (imaginary parts of the eigenvalues) and the traveling speed of the string. The solid and the dashed lines represent the natural frequencies of the coupled system with $m_{2}=0.2$ and 2 , respectively, at position $\xi=0.5$. The frequencies of the freely traveling string are presented as dotted lines for comparison. The number of modes $N$ used in the eigenfunction expansion (10) is 6 . The accuracy and convergence of the numerical results are examined by comparing the calculated eigenvalues with those obtained by taking $N=12$. For simplicity and clarity, however, only the eigenvalues of the modes with $n \leq 3$ are shown here. It is observed in Fig. 2 that the added inertia in the stationary load system tends to decrease the natural frequencies of all modes, unless the added mass happens to be at the nodal point of the stationary envelope of a particular mode. The real parts of all the eigenvalues are not affected by the added mass and always remain zero. In other words, inertia in the stationary load system does not affect the stability of the system.

The solid and the dashed lines in Fig. 3 represent the natural frequencies of the coupled system with $k_{z}=20$ and 1000 , respectively, at position $\xi=0.3$. Dotted lines represent the natural frequencies of the freely traveling string. Apparently, the effects of $k_{z}$ are exactly opposite to those of $m_{z}$. The solid, dashed, and dotted lines in Fig. 4 are the envelopes of the mode shapes of the first three modes, with natural frequencies $1.59,3.16$, and 3.64 respectively, when the string is traveling at $\nu=0.8$ and is constrained by $k_{z}=20$ at $\xi=0.3$. It can be seen that the vibrations are localized in the right section of the string for the first two modes, and in the left section for the third mode. As $k_{z}$ approaches infinite, the vibrations in the left span of the first two modes and the right span of the third mode diminish, and the corresponding natural frequencies approach $n \pi\left(1-\nu^{2}\right) /$ $(1-\xi), n=1,2$, and $\pi\left(1-\nu^{2}\right) / \xi$, respectively. These properties are in agreement with the expectation that the left span and

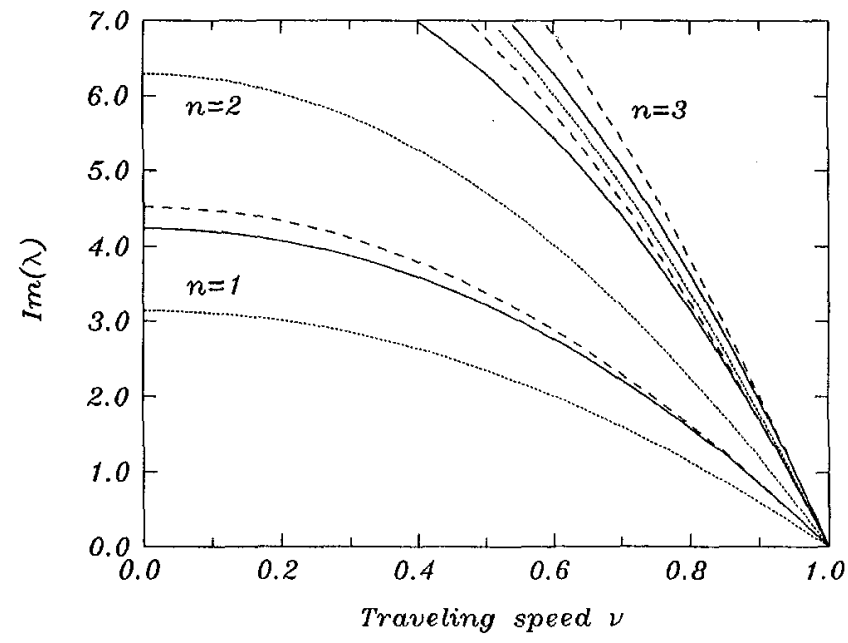

Fig. 3 Effects of stiffness $k_{z}=20$ (solid lines) and 1000 (dashed lines) at $\xi=0.3$ on the natural frequencies of the traveling string, compared to the results for a freely traveling string (dotted lines). All other parameters are zero. 


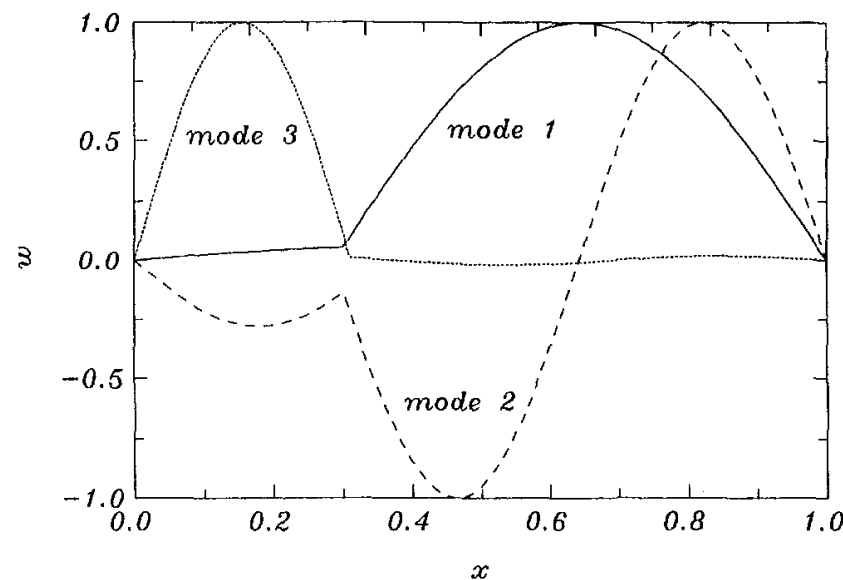

Fig. 4 Envelopes of mode shapes for the first three modes of the string traveling at $\nu=0.8$ and constrained by a spring $k_{2}=20$ at $\xi=0.3$

the right span of the traveling string are decoupled into two independent sections with the same tension.

It is of interest to examine the combined effects of inertia and stiffness in the load system. The solid lines in Fig. 5 represent the results for a coupled system with $m_{z}=0.2$ and $k_{z}=3$ at position $\xi=0.3$. The corresponding natural frequency in the load system is $\omega_{z}=\sqrt{k_{z} / m_{z}}=3.87$. The dotted lines represent the frequencies of the freely traveling string again. It is found that for the modes with $\omega_{n}>3.87$, the inertia effect is dominant. For the modes with $\omega_{n}<3.87$, on the other hand, the stiffness effect is dominant. The eigenvalues of the modes with natural frequencies exactly equal to the natural frequency of the load system will not be affected by the addition of the load system.

Figure 6 shows the effects of damping $c_{z}=1$ at position $\xi$ $=0.3$ on the eigenvalues of the system. It is observed that damping in the stationary load system tends to stabilize all the modes in the sub-critical speed range. The effect of damping on the natural frequencies is minimal.

When the traveling string is subjected to a large friction force $f_{\theta}=0.75$ and is constrained by a spring $k_{z}=20$ at position $\xi$ $=0.5$, Cheng and Perkins (1991) reported that flutter instability was induced for traveling speed $\nu$ higher than 0.5 , as shown in the middle and the lower graphs of Fig. 7. The string in this case can be divided into two sections; the tension in section 0 $<\xi<0.5$ is 0.25 , while the tension in section $0.5<\xi<1$

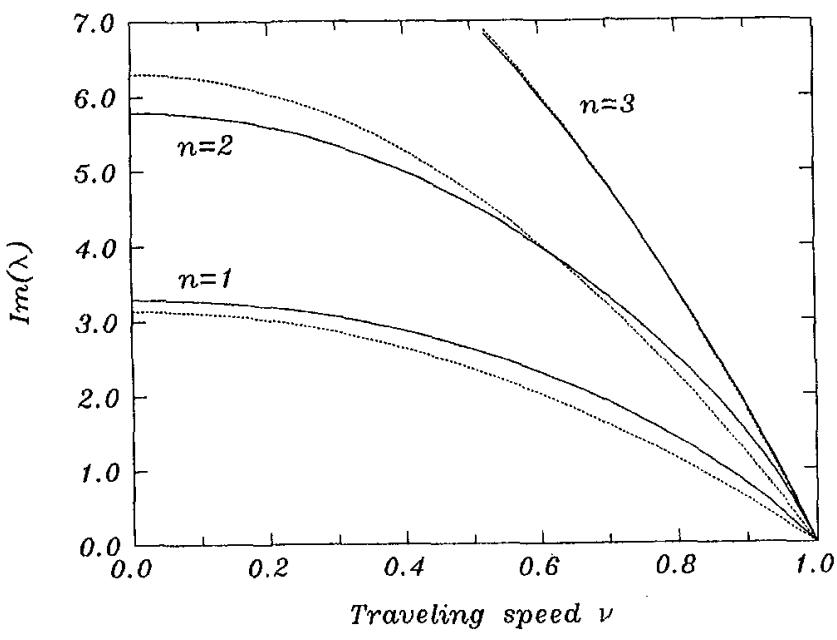

Fig. 5 Combined effects of $m_{z}=0.2$ and $k_{z}=3$ at $\xi=0.3$ on the natura frequencies of the traveling string, compared to the results for a freely traveling string (dotted lines)
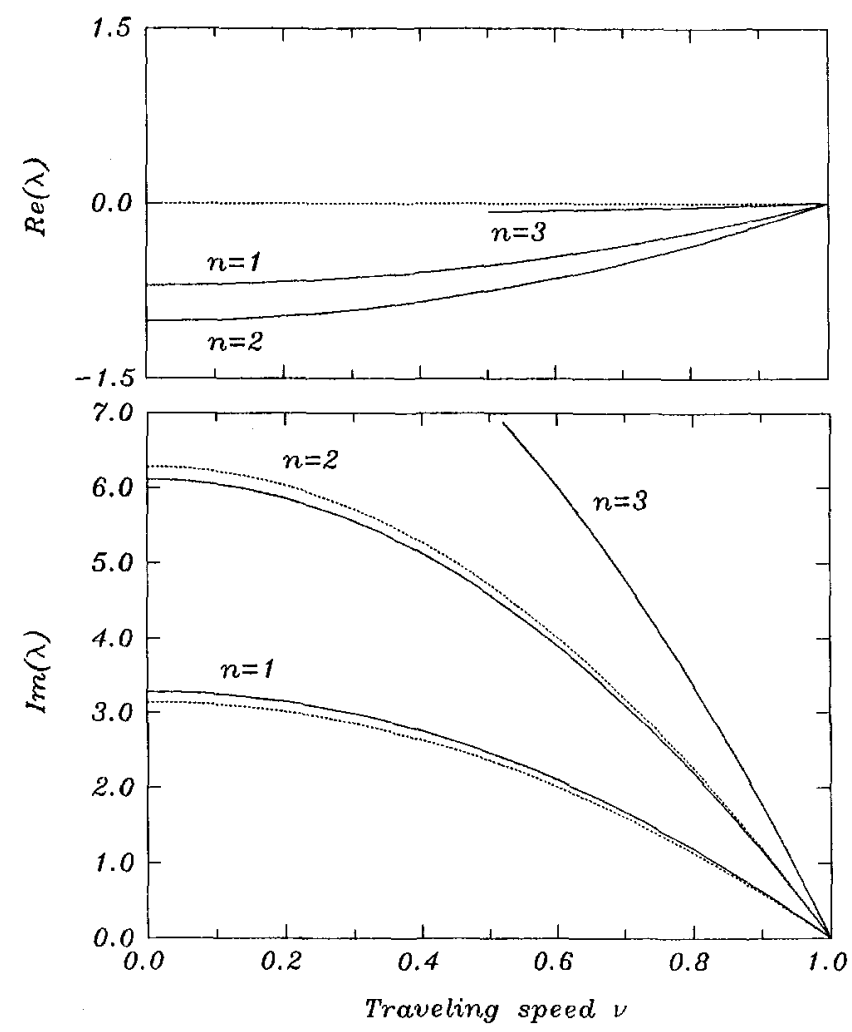

Fig. 6 Effects of damping $c_{x}=1$ at $\xi=0.3$ on the eigenvalues of the system, compared to the results for a freely traveling string (dotted lines). All other parameters are zero.

remains 1 . When the translating speed $\nu>0.5$, it is beyond the critical speed for the section $0<\xi<0.5$, and flutter instability is induced. In the low speed range, Cheng and Perkins (1991) observed frequency loci veering and the associated mode localization phenomena. Since damping alone can stabilize the traveling string, it is of interest to see whether damping can suppress the instability induced by large friction force. The upper graph of Fig. 6 shows the real parts of the eigenvalues for a traveling string subject to tension $f_{\theta}=0.75, k_{z}=20$, and damping $c_{z}=1$ at position $\xi=0.5$. The natural frequencies are very close to the case without damping. It is noted that while damping can indeed stabilize all modes when $\nu<0.5$, it cannot suppress the flutter instability induced by large friction. A careful inspection on the eigenvalue loci reveals that the modes whose natural frequencies increase with traveling speed (i.e., the modes traveling downstream) are destabilized, in spite of the fact that they are originally stable when damping is absent.

\section{Derivatives of Eigenvalues}

While the freely traveling string is a standard gyroscopic system and all the eigenvalues are purely imaginary, the stringload system is not necessarily gyroscopic or stable. The matrix differential operators $\hat{\mathbf{A}}$ and $\hat{\mathbf{B}}$ in Eq. (3) are functions of certain parameters $\rho_{k}(k=1,2, \ldots, l)$ in the stationary load system. It was shown in Chen and Bogy (1992) that expressions for the derivatives of the eigenvalues with respect to various parameters in the stationary load system, which renders the system non-gyroscopic, can be derived and used to predict the change of eigenvalues for small values of the parameters. The first order derivative of the eigenvalue $\lambda_{n}$ with respect to $\rho_{k}$ can be calculated by the formula 

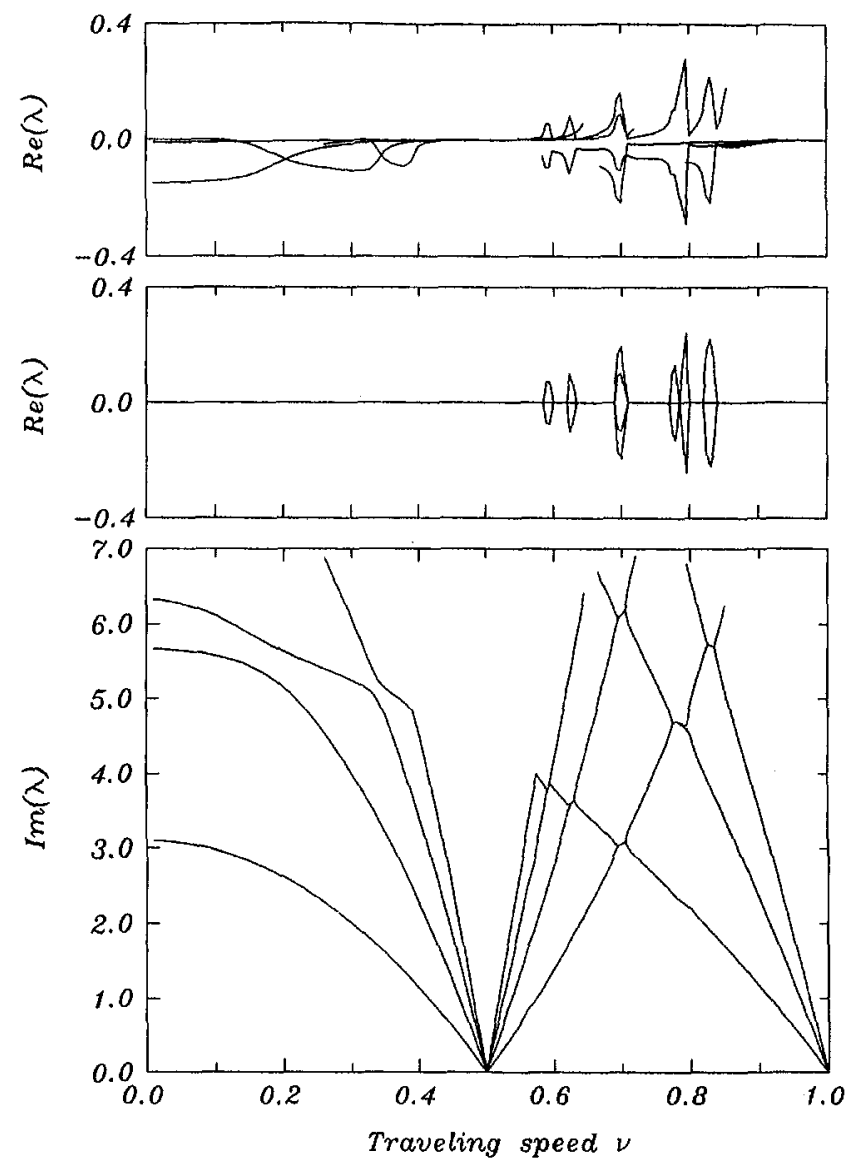

Fig. 7 The middle and the lower graphs show the eigenvalues of the system with $f_{\theta}=0.75$ and $k_{x}=20$ at position $\xi=0.25$. The upper graph shows the real parts of the eigenvalues when additional damping $c_{z}=1$ is introduced.

$$
\frac{\partial \lambda_{n}}{\partial \rho_{k}}=-\frac{\left\langle\mathbf{x}_{n}^{0},\left(\lambda_{n}^{0} \hat{\mathbf{A}}_{, k}-\hat{\mathbf{B}}_{, k}\right) \mathbf{x}_{n}^{0}\right\rangle}{\left\langle\mathbf{x}_{n}^{0}, \mathbf{A} \mathbf{x}_{n}^{0}\right\rangle}
$$

The change of eigenvalue $\Delta \lambda_{n}$ corresponding to a set of parametric increments $\Delta \rho_{k}$ can be approximated as

$$
\Delta \lambda_{n}=\sum_{k=1}^{l} \lambda_{n, k} \Delta \rho_{k}
$$

Inertia Effect. After deriving $\partial \hat{\mathbf{A}} / \partial m_{z}$ and $\partial \hat{\mathbf{B}} / \partial m_{z}$ and substituting the results into Eq. (12), with the expressions of eigenvalue $\lambda_{n}^{0}$ in Eq. (5) and eigenfunction $w_{n}^{0}$ in Eq. (6), we obtain

$$
\frac{\partial \lambda_{n}}{\partial m_{z}}=-i n \pi\left(1-\nu^{2}\right)^{2} \sin ^{2} n \pi \xi
$$

Equation (14) predicts that adding inertia in the load system tends to decrease the natural frequencies of the system. In addition, the derivative vanishes whenever $n \xi$ equals to an integer. For $\xi=0.5$, for instance, the natural frequencies of the modes with $n=2,4,6, \ldots$ will not be affected by adding $m_{z}$ in the load system.

Stiffness Effect. By following a similar procedure as above, we can get

$$
\frac{\partial \lambda_{n}}{\partial k_{z}}=\frac{i \sin ^{2} n \pi \xi}{n \pi}
$$

Clearly, adding a weak spring tends to increase the natural frequencies of the system in the sub-critical speed range.
Suppose the load system contains both $m_{z}$ and $k_{z}$ with natural frequency $\omega_{z}=\sqrt{k_{z} / m_{z}}$. By applying Eq. (13) we obtain

$$
\Delta \lambda_{n}=\frac{i m_{z}}{n \pi}\left(\omega_{z}^{2}-\omega_{n}^{2}\right) \sin ^{2} n \pi \xi
$$

In the sub-critical speed range, the load system tends to increase the natural frequencies of the modes with $\omega_{n}<\omega_{2}$, and tends to decrease the natural frequencies of the modes with $\omega_{n}>\omega_{z}$. Therefore, the stiffness effect is dominant for $\omega_{n}<\omega_{z}$, and inertia effect is dominant for $\omega_{n}>\omega_{z}$. The natural frequencies of the modes with $\omega_{n}=\omega_{z}$ will not be affected by this load system. This phenomenon may also be predicted by an eigenvalue inclusion principle for distributed gyroscopic systems proposed by Yang (1992).

Damping Effect. For the case of damping

$$
\frac{\partial \lambda_{n}}{\partial c_{z}}=-\left(1-\nu^{2}\right) \sin ^{2} n \pi \xi
$$

Note that this derivative is real and the dashpot will affect the stability of the system instead of the natural frequency. Since this derivative is negative, the dashpot tends to stabilize the system in the sub-critical speed range. On the other hand, damping tends to destabilize the system in the supercritical speed range.

Friction Effect. As we have discussed in the preceding section that friction force affects the natural frequencies and stability of the traveling string through two avenues. One is the term associated with Dirac delta function and the other is the term associated with Heaviside function in the differential operator $\hat{K}$. The effects of these two terms on the eigenvalues of the system can be predicted by the following two formulae,

$$
\left.\frac{\partial \lambda_{n}}{\partial f_{\theta}}\right|_{\delta}=-\frac{i}{2} \sin 2 n \pi \xi+\nu \sin ^{2} n \pi \xi
$$

$\left.\frac{\partial \lambda_{n}}{\partial f_{\theta}}\right|_{H}=\frac{i}{4}\left(3+\nu^{2}\right)[-2 n \pi \xi+\sin 2 n \pi \xi]-\nu \sin ^{2} n \pi \xi$

where subscripts $\delta$ and $H$ represent the effects of the terms with delta function and Heaviside function, respectively. It is noted by observing Eqs. (16) and (17) that transverse component of the dry friction associated with slope change tends to destabilize the system, while the term associated with tension variation tends to stabilize it. These two opposite effects cancel and as a consequence the total effect of the friction force is to change the natural frequency of the string only. It is noted that this conclusion is good only when small friction is involved.

\section{Conclusions and Discussions}

The natural frequencies and stability of an axially-traveling string in contact with a stationary load system containing such parameters as friction force, inertia, damping, and stiffness are investigated both numerically and analytically. The results of these analyses can be summarized as follows:

(1) The inertia in the stationary load system tends to decrease the natural frequencies of all modes of the system in the sub-critical speed range, unless the added mass happens to be at the nodal point of the stationary envelope of a particular mode. The effects of stiffness are opposite to those of the inertia.

(2) When the load system contains both inertia and stiffness elements simultaneously with natural frequency $\omega_{z}$, inertia effect is dominant for the modes with natural frequencies higher than $\omega_{z}$. On the other hand, stiffness effect is dominant for the modes with natural frequencies lower than $\omega_{z}$. 
(3) The damping element in the load system tends to stabilize the system in the sub-critical speed range.

(4) Large dry friction induces flutter instability in the high traveling speed range. It is impossible to suppress these unstable vibrations by a damping element in the stationary load system.

The traveling string may be the simplest model for an axiallymoving structure, partly because it is non-dispersive. For many one-dimensional structure, however, the bending effect cannot be neglected. A natural extension of this paper is to study the dynamics of a traveling beam in contact with a stationary load system. It is believed that the concepts presented in this paper are valuable in studying more complex problems.

\section{References}

Archibald, F. R., and Emslie, A. G., "The Vibration of a String Having a Uniform Motion Along Its Length," ASME Journal of Applied Mechanics, Vol. 25 , pp. $347-348,1958$

Chen, J.-S., and Bogy, D. B., "Effects of Load Parameters on the Natural Frequencies and Stability of a Flexible Spinning Disk With a Stationary Load System," ASME Journal of Applied Mechanics, Vol. 59, Part 2, pp. 230-235, 1992.

Cheng, S.-P., and Perkins, N. C., "The Vibration and Stability of a FrictionGuided, Translating String," Journal of Sound and Vibration, Vol. 144, No. 2 pp. 281-292, 1991

D'Eleuterio, G. M. T., and Hughes, P. C., "Dynamics of Gyroelastic Continua," ASME Journal of Applied Mechanics, Vol. 51, pp. 415-422, 1984
Hwang, S.-J., and Perkins, N. C., "Supercritical Stability of an Axially Moving Beam. Part I: Model and Equilibrium Analysis," Journal of Sound and Vibration, Vol. 154, pp. 381-396, 1992a.

Hwang, S.-J., and Perkins, N. C., "Supercritical Stability of an Axially Moving Beam. Part II: Vibration and Stability Analysis," Journal of Sound and Vibration, Vol. 154, pp. 397-409, 1992b.

Mahalingam, S., "Transverse Vibrations of Power Transmission Chains," British Journal of Applied Physics, Vol. 8, pp. 145-148, 1957.

Meirovitch, L., "A New Method of Solution of the Eigenvalue Problem for Gyroscopic Systems," AIAA Journal, Vol. 12, pp. 1337-1342, 1974

Miranker, W. L., "The Wave Equation in a Medium in Motion," IBM Journal of Research and Development, Vol. 4, pp. 36-42, 1960.

Rhodes, J. E., Jr., "Parametric Self-Excitation of a Belt into Transverse Vibration," ASME Journal of Applied Mechanics, Vol. 37, pp. 1055-1060, 1970.

Sack, R. A., "Transverse Oscillations in Traveling Strings," British Journal of Applied Physics, Vol. 5, pp. 224-226, 1954.

Schajer, G. S., "The Vibration of a Rotating Circular String Subject to a Fixed Elastic Restraint," Journal of Sound and Vibration, Vol. 92, pp. 11-19, 1984.

Skutch, R., "Uber die Bewegung Eines Gespannten Fadens, Weicher Gezwungen ist, Durch Zwei, Feste Punkte, mit Einer Constanten Geschwindigkeit zu gehen, und Zwischen denseiben in Transversal-Schwingungen von gerlinger Amplitude Versetzt Wird," Annalen der Physik und Chemie, Vol, 61, pp. 190$195,1897$.

Swope, R. D., and Ames, W. F., "Vibrations of a Moving Threadline," Journal of Franklin Institute, Vol. 275, pp. 36-55, 1963

Wickert, J. A., and Mote, C. D., Jr., "Current Research on the Vibration and Stability of Axially-Moving Materials," Shock and Vibration Digest, Vol. 20, pp. 3-13, 1988.

Wickert, J. A., and Mote, C. D., Jr., "Classical Vibration Analysis of AxiallyMoving Continua," ASME Journal of Applied Mechanics, Vol. 57, pp. 738744,1990 .

Yang, B., "Eigenvalue Inclusion Principles for Distributed Gyroscopic Systems," ASME Journal of Applied Mechanics, Vol. 59, pp. 650-656, 1992. 